Rhode Island College

Digital Commons @ RIC

Master's Theses, Dissertations, Graduate

Master's Theses, Dissertations, Graduate Research and Major Papers Overview

Research and Major Papers

$12-11-2018$

\title{
Evaluation of a Perinatal Human Papillomavirus Program
}

Melissa J. Maher

Follow this and additional works at: https://digitalcommons.ric.edu/etd

Part of the Nursing Commons

\section{Recommended Citation}

Maher, Melissa J., "Evaluation of a Perinatal Human Papillomavirus Program" (2018). Master's Theses, Dissertations, Graduate Research and Major Papers Overview. 281.

https://digitalcommons.ric.edu/etd/281

This Thesis is brought to you for free and open access by the Master's Theses, Dissertations, Graduate Research and Major Papers at Digital Commons @ RIC. It has been accepted for inclusion in Master's Theses, Dissertations, Graduate Research and Major Papers Overview by an authorized administrator of Digital Commons @ RIC. For more information, please contact digitalcommons@ric.edu. 


\title{
EVALUATION OF A PERINATAL
}

\section{HUMAN PAPILLOMAVIRUS VACCINATION PROGRAM}

\author{
by \\ Melissa J. Maher \\ A Major Paper Submitted in Partial Fulfillment \\ of the Requirements for the Degree of \\ Master of Science in Nursing \\ in \\ The School of Nursing \\ Rhode Island College \\ 2018
}




\begin{abstract}
Human papillomavirus (HPV) is recognized as the most prevalent sexually transmitted infection in the United States. Prevention of HPV infection is possible with the efficacy of HPV vaccine noted to be nearly $100 \%$ when introduced prior to sexual debut. In addition, vaccination post-sexual initiation could have a significant impact on reducing HPV infection and HPV-associated cancers in women. Much focus has been placed on vaccinating adolescents against HPV. However, strategies to increase vaccination rates among females ages 18-26, who remain eligible for vaccination, must be examined. A program evaluation was undertaken to assess the effectiveness of a newly developed pilot program for women ages 19-26 that incorporates assessment of vaccine eligibility during the prenatal period to increase uptake and completion of HPV vaccine postpartum. Data collected from the perinatal vaccination pilot program was performed retrospectively. Through a chart review, postpartum patients age 19-26 were identified and specific areas of focus were examined: whether an HPV dose was administered to vaccine eligible individuals during the inpatient period; whether an additional dose was administered at an ambulatory postpartum visit; and whether a subsequent dose was given at an ambulatory visit if warranted. Data analysis was conducted. Frequencies and percentages in relation to numbers of patients and HPV doses were calculated. Finally, implications for advanced nursing practice were discussed.
\end{abstract}




\section{Acknowledgements}

I would like to thank Dr. Byrd, Dr. Costello, and Dr. Blanchette for their time, patience, and unwavering support of this master's project. I would additionally like to thank Dr. Beth Cronin, Denise Henry RN, and Dr. Lauren Thorngate for encouraging me to pursue the development and evaluation of this pilot program. Finally, I would also like to acknowledge my husband, Will, and my children, Meaghan and Sean. They have been my supportive champions and without them I would have never completed this journey. 


\section{Table of Contents}

Background/Statement of the Problem ....................................................................... 1

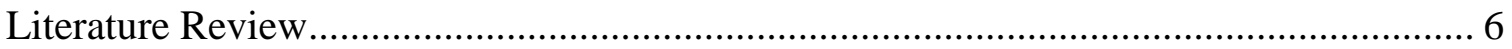

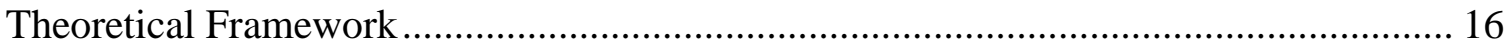

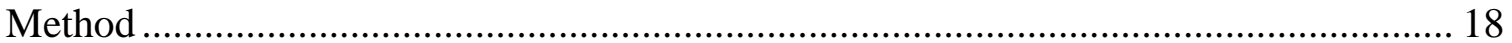

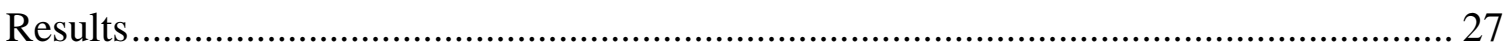

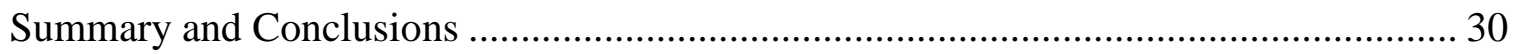

Recommendations and Implications for Advanced Nursing Practice ............................ 34

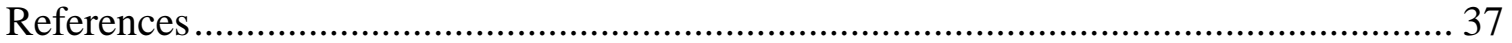

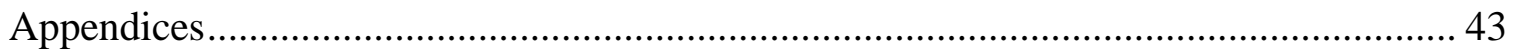


Evaluation of a Perinatal Human Papillomavirus Vaccination Program

\section{Background/Statement of the Problem}

Human papillomavirus (HPV) is recognized as the most prevalent sexually transmitted infection in the United States, accounting for 39,000 cases of HPV-related cancers including those of the cervix, vulva, vagina, penis, oropharynx, and anus (Petrosky et al., 2015; Saraiya et al., 2015; Centers for Disease Control, 2017). Approximately 100 identified strains of HPV have been identified including 40 high-risk, oncogenic strains (CDC, 2017). HPV is classified as oncogenic and non-oncogenic with the highest incidence of cancers resulting from strains 16 and 18 (CDC, 2017). HPV also accounts for the majority of cases of cervical dysplasia, a pre-cancerous condition of cervix. Cervical dysplasia has both a global financial impact as well as a significant impact on quality of life for those affected (Handler, Handler, Majewski, \& Schwartz, 2015; Petrosky et al., 2015). Notably, though not cancer causing, non-oncogenic strains of HPV have a significant disease burden as evidenced by the incidence of genital condyloma.

Quadrivalent-HPV vaccine (4vHPV) was introduced in 2006 as the first Federal Drug Administration (FDA) approved vaccine, Gardasil, targeting HPV strains 16, 18, 6, and 11, followed by the 2009 FDA approved bivalent-HPV vaccine (2vHPV), Cervarix, for strains 16 and 18, and the 2015 introduction of nonavalent HPV vaccine (9vHPV), Gardasil9, which included the 4vHPV strains and strains 31, 33, 45, 52, and 58, an additional five oncogenic-associated strains (Handler et al., 2015; Petrosky et al., 2015). Initial recommendations by the Advisory Committee on Immunization Practices (ACIP) focused on vaccinating females ages 11-26 for 4vHPV and 2vHPV with 
recommendations now in place for males ages $11-26$ to receive 4vHPV (Petrosky et al., 2015). Additionally, 9vHPV can be administered as early as age 9, to all females ages 1126 and males age 11-21, and up to age 26 for men who have sex with men or men who have sex with women and men (Petrosky et al., 2015).

The efficacy of 4vHPV and 9vHPV in preventing strains 16, 18, 6, and 11 has been noted to be nearly $100 \%$ when introduced prior to sexual debut with clinical trials showing a nearly 97\% reduction in disease associated with HPV strains 31, 33, 45, 52, and 58\% reduction for those participants who received 9vHPV versus 4vHPV vaccine (Joura et al., 2015). Introduction of HPV vaccination prior to initiation of sexual activity is ideal. However, especially with 9vHPV, which provides vaccine coverage for an additional five HPV strains that are associated with $18.5 \%$ of cervical cancers, the introduction of this vaccine series post-sexual initiation could still have a significant impact on reducing HPV-associated cancers in women (Serrano et al, 2012). Vaccination post-sexual initiation is beneficial as it offers protection against strains that may have not been previously acquired (Serrano et al., 2012).

Despite evidence to support high levels of efficacy in preventing HPV-associated dysplasia and cancers with HPV vaccination, the rates of vaccination in the United States for adolescents ages 13-17 remain low with only 63\% of females and 50\% of males having received at least one dose of HPV vaccine (Reagan-Steiner et al., 2016). The evidence identifies a gap in which there is opportunity to initiate or continue the HPV series for those ages 18-26 (Reagan-Steiner et al., 2016).

The female population, whose risk of disease burden is greatly impacted by HPV, is of significant priority. With one in four Americans at-risk for developing HPV 
infection and $90 \%$ of cervical cancers attributed to HPV, prevention of HPV infection must be emphasized (Serrano et al., 2012; CDC, 2017). The United Nations' focus on improving the health of the population through the Sustainable Development Goals (SDG) recognizes the impact that women's health has on society. Prevention of HPVassociated morbidity and mortality in the female population supports the prospect of a healthier community. Goals three through five of the SDGs apply to HPV prevention. The United Nations (2015) identifies these women's health SDG goals as:

Goal 3. Ensure healthy lives and promote well-being for all at all ages; Goal 4. Ensure inclusive and equitable quality education and promote lifelong learning opportunities for all; and Goal 5. Achieve gender equality and empower all women and girls (p. 18-20).

In developing countries, HPV vaccination rates have yet to reach the same levels as higher income countries (World Health Organization, 2016). However, with the SDGs, through health promotion, education, and empowerment, the opportunity to reduce HPV infection or even eradicate cervical cancer for future generations,' remains a possibility. HPV vaccination rates in the United States remain sub-optimal with only 34.9\% of all adolescents ages 13-17 having received the complete three-dose series of HPV vaccine (Reagan-Steiner et al., 2016). In a review of the data from the National Immunization Survey-Teen between 2008 to 2013, HPV vaccination initiation and series completion among various racial and ethnic groups was examined (Burdette, Webb, Hill, \& Jokinen-Gordon, 2017). Findings show that by 2013, initiation of the vaccine series increased for all racial and ethnic groups with higher rates of initiation by AfricanAmerican and Hispanic women as compared to non-Hispanic white women (Burdette et 
al., 2017). Additionally, the likelihood of HPV vaccine series completion was greatest among Hispanic females as compared to non-Hispanic white females (Burdette et al., 2017). Provider recommendation to vaccinate also showed an increase among all racial and ethnic groups, with a 19\% increase yearly (Burdette et al., 2017). However, the likelihood of receiving a recommendation to vaccinate was higher among AfricanAmerican and Hispanic females (Burdette et al., 2017).

Healthy People 2020 data indicates that HPV vaccination rate disparities continue to exist for those ages 13-15 among ethnic groups and household income levels (U. S. Department of Health and Human Service, 2017). From 2012 to 2015, data demonstrate that a decrease has occured in adolescent females ages 13-15 years who identify as whiteonly in receiving three doses of the HPV series (U.S. DHHS, 2017). Additionally, females ages 13-15 years whose mothers have a four-year college degree and a household income level at or above the poverty level are less likely to receive HPV vaccination (U.S. DHHS, 2017). Hispanic and black females, those whose mothers have less than a high school education, and those who live below the federal poverty level have greater levels of three dose HPV series completion (U.S. DHHS, 2017).

Though much research has emphasized vaccination programs for adolescents, less focus has been placed on older-adolescents and young adults who are at-risk for HPV infection and remain eligible for vaccination. In 2012, the overall HPV vaccination rate through three doses of the series for girls age 13-15 was only 28.1 percent (U. S. DHHS, 2017). This cohort of the older adolescent and young-adult subset, now ages 18-20, are at great risk for HPV infection due to low rates of vaccine acceptance as youngeradolescents. In response, Healthy People 2020 has identified the need to address this risk 
by including the developmental objective of reducing the overall proportion of females with HPV infection (U.S. DHHS, 2017). Few studies have looked at whether primary prevention strategies result in an increase in HPV vaccination rates in this population of females ages 18-26.

Strategies to increase vaccination rates among females ages 18-26 must be examined. With increased age, the likelihood of sexual activity and childbearing increases among this population. Pregnancy is a period when health promotion and access to health care becomes of significant importance. In Rhode Island, a Medicaid expansion state, uninsured pregnant women qualify for Medicaid if they are within 253\% of the federal poverty level (Rhode Island Health \& Human Services, 2017). While HPV vaccination is currently not recommended during pregnancy, assessment of HPV vaccination status should be included during prenatal care. Protocols for vaccinating women in the postpartum period should be explored. Educating women prenatally about HPV virus and their vaccine eligibility in the immediate postpartum period are of equal importance as individual health promotion activities may become displaced by a woman's focus on infant care once hospital discharge has occurred. The implementation of a perinatal program that includes postpartum vaccination may increase the overall vaccination rates for women ages 19-26 and needs to be further explored. 


\section{Literature Review}

The purpose of this literature review was to identify common themes pertaining to human papilloma virus, HPV vaccination, and postpartum HPV vaccination. A search was conducted using CINAHL Plus with full text, PubMed Central, and Google. Search terms included human papilloma virus, HPV, vaccine, incidence, prevalence, efficacy, immunogenicity, rates, female, and postpartum. The reference lists of identified articles were reviewed for additional sources. The publication dates were limited to the time frame from 2006 through 2018.

\section{HPV Incidence and Prevalence}

Globally, HPV is a predominant health problem with the World Health Organization (WHO) estimating 528,000 new cases of HPV in 2012 (WHO, 2017).

Though many who seroconvert to an HPV-positive status have a transient viral infection, many others have persistent virus that can result in dysplasia or oncogenic forms of the disease (Braaten \& Laufer, 2008). In the United States, HPV is the most prevalent sexually transmitted infection with an estimated $25 \%$ of the population having HPV infection during their lifetime (CDC, 2017).

CDC (2017) cancer registry data attributes 39,844 cancers to HPV which dramatically demonstrates the magnitude of the impact that HPV has on U.S. society. However, the global impact of HPV incidence and prevalence must be reviewed to understand the effect on the world's population. Universally, the body of literature regarding HPV identifies HPV as the causative agent in greater than $90 \%$ of cervical cancers with HPV strains 16 and 18 identified in greater than 70\% of those cases (Serrano et al., 2012; Saraiya et al., 2015; CDC, 2017). 
In the review of the literature by Serrano et al. (2012), the researchers examined two international studies to determine the relative correlation (RC) of HPV-types included in the 9vHPV vaccine to overall invasive cervical carcinoma rates and precancerous cervical lesions. The researchers examined a retrospective cross-sectional study of 10,575 invasive cervical cancer cases between 1949 and 2009 from 38 countries with 8,977 HPV-positive cases (de Sanjose et al., 2010; Serrano et al., 2012). Archived pathological samples were analyzed to determine the prevalence of each HPV-type attributed to cervical cancer cases in subjects classified by 10 geographical regions and by age-group (de Sanjose et al., 2010; Serrano et al., 2012). Additionally, data from an International Agency for Research on Cancer meta-analysis was reviewed to examine the distribution of HPV types among 115,789 HPV-positive women from 423 studies (Serrano et al., 2012). The researchers concluded that $89.4 \%$ of invasive cervical cancers had an RC to one of the 9 HPV-types included in the 9vHPV vaccine (Serrano et al., 2012). Additionally, the RC of the 9 HPV-types to pre-cancerous diagnosis of squamous cell carcinoma was $89.1 \%$ and $95.5 \%$ for adenocarcinoma (Serrano et al., 2012). Using calculated data for 2012 and projected data for 2025 based upon global population growth and current HPV rates, de Sanjose et al. (2010) suggest, that without HPV vaccination, global cases of invasive cervical cancer could rise to greater than 627,000 new cases in 2025. Serrano et al. (2012) theorize that greater access to 9vHPV vaccine, especially in developing regions of the world where cervical cancer screening programs are inconsistent or non-existent, could have a greater proportional impact by reducing HPV rates through immunity, therefore reducing overall invasive cancer rates and HPVrelated deaths. 
In the US population, HPV presents a significant threat in terms of morbidity and mortality. In the research by Saraiya et al. (2015), CDC Cancer Registry Sentinel Surveillance System data was analyzed to determine the prevalence and types of HPVassociated cancers in the U.S. pre-HPV vaccine implementation. Though the clinical correlation between HPV and cervical cancer is widely accepted, the researchers expanded their focus to examine additional types of HPV-associated cancers including those of the anus, vagina, oropharynx, vulva, penis, oral cavity, and larynx (Saraiya et al, 2015). CDC cancer registry data was examined from 1993 to 2005 with 2,670 tissue samples representative of seven areas of the country that have high-rates of cervical cancer compared to other areas of the U.S. (Saraiya et al., 2015). The researchers concluded that HPV presence was highest in cases of cervical cancer in-situ (98.8\%), cervical (90.6\%), anal (91.1\%), penile (63.3\%), and oropharyngeal cancers $(70.1 \%)$ (Saraiya et al., 2015). The presence of HPV-16 was highest in all types of HPVassociated cancers (Saraiya et al., 2015). Based upon these conclusions, the researchers hypothesize that if the U.S. population was fully vaccinated against HPV, greater than 24,000 HPV-associated cancers could be prevented yearly (Saraiya et al., 2015).

\section{HPV Vaccination Efficacy and Immunogenicity}

The impact of HPV upon global society and the U.S. population is notable. With over a decade of HPV vaccination programs using 4vHPV and bivalent-HPV vaccine, efficacy and immune response has been well established (Joura et al., 2015). The overall effectiveness and immune response to 9vHPV vaccine clearly demonstrates the shortterm benefit of vaccination programs. With the introduction of 4vHPV vaccination in 2006, ACIP adopted recommendations for routine HPV vaccinations for specific patient 
populations (Petrosky et al., 2015). With the introduction of 9vHPV vaccine, evolving research on vaccine efficacy and immunogenicity has emerged. In response to this research, both ACIP in the U.S. and the Global Alliance for Vaccines and Immunization (GAVI) have expanded vaccine coverage for both the male and female populations in the U.S. and in developing regions of the world where it is estimated that by 2020 fortymillion girls could be protected against cervical cancer by receiving HPV vaccination (Petrosky et al., 2015; GAVI, 2017).

In clinical trials of the 9vHPV vaccine, researchers conducted a randomized, international, double-blind study consisting of 14,215 participants ages 16-26 randomly assigned to receive either 4vHPV, Gardasil, or 9vHPV, Gardasil 9, vaccine (Joura et al., 2015). Participants were followed to observe if HPV vaccination offered risk reduction in developing disease related to HPV in either vaccine type (Joura et al., 2015). Results showed that Gardasil 9 was $96.7 \%$ effective in reducing cases of cervical, vulvar, and vaginal disease or cancer attributable to HPV types 31, 33, 45, 52, and 58 and that clinical effectiveness was comparable to 4vHPV in reducing HPV 16 and 18 (Joura et al., 2015). Immunogenicity was also examined and showed that within the control group nearly $100 \%$ of participants seroconverted to the 9vHPV vaccine types and that geometric mean titers showed immune responses for HPV-6, 11, 16, 18 to be comparable to quadrivalent vaccine (Joura et al., 2015). The results of this efficacy and immunogenicity study showing that the 9vHPV vaccine is non-inferior to quadrivalent vaccine has led to the 9vHPV vaccine being accepted as the standard HPV vaccine option. 
With the 9vHPV introduction in 2015, further studies have resulted in continued revision of dosing schedules based upon immunogenicity. In December 2016, ACIP modified its 9vHPV dosing recommendations based upon clinical trials that examined the immune response to a 2-dose vaccine schedule for those ages 9-14 as compared to the traditional 3-dose regimen in those ages 16-26. Study participants consisted of 1,518 boys and girls from 15 countries divided into five cohorts to examine the immune response of varying dosing schedules as compared to the control group (Iverson et al., 2016). The results showed that the geometric mean titer at four weeks post vaccination for the twodose schedule, given at day one and month six, were non-inferior to the geometric mean titers for traditional three-dose vaccination (Iverson et al., 2016).

Prior to the aforementioned clinical trial, researchers had examined the immunogenicity and efficacy of receiving one, two, or three doses of the 4vHPV vaccine. In a prospective cohort study of 17,729 girls' similar findings were noted in that participants who received two-doses at day one and month six had non-inferior geometric mean titers as compared to the three-dose cohort (Sankaranarayanan et al., 2016). Additionally, those who received only one dose also had detectable levels of HPV antibodies, but at lower concentrations (Sankaranarayanan et al., 2016). This could lead to the conclusion that those who had one dose would have a higher level of immunity to HPV as compared to natural immunity. Cervical samples from 2,649 participants were obtained to evaluate the efficacy of one, two, or three dose 4vHPV vaccine (Sankaranarayanan et al., 2016). Remarkably, the overall incidence of HPV strains 6, 11, 16, and 18 detected in cervical samples were comparable for those participants who received one, two, or three doses of vaccine (Sankaranarayanan et al., 2016). The 
researchers concluded that the two-dose vaccination schedule could have a significant impact on reducing global cervical cancer rates in low to middle income countries where adherence to the vaccine schedule is less successful than higher income countries and that more research needs to be done examining one-dose vaccine programs (Sankaranarayanan et al., 2016).

\section{HPV Vaccine Rates}

Though evidence exists to support alternative dosing schedules and ACIP guidelines recommend the two-dose schedule for those ages 9-14, overall vaccine rates should be examined to identify if gaps in meeting the three-dose recommendation exist in other vaccine eligible populations (Iverson et al., 2016; Meites, Kempe, \& Markowitz, 2016; Sankaranarayanan et al., 2016). In a review of 21,875 participants of the 2015 National Immunization Survey-Teen (NIS-Teen), data regarding HPV vaccination among teens both male and female ages 13-17 years was obtained (Reagan-Steiner, 2016). A review of provider reported vaccine histories showed only $39.4 \%$ of those vaccinated had received three doses of HPV vaccine (Reagan-Steiner et al., 2016). Many participants initiated the series, $56.1 \%$, but the percentage of those who continued with the series began to lag with dose two at 45.4\%. (Reagan-Steiner et al., 2016). The researchers suggest that HPV vaccination rates remain low due to inconsistent recommendations by providers, lack of co-administration of HPV vaccine with other childhood immunizations, and misinterpretation of risk exposure and parental concerns (Reagan-Steiner et al., 2016). Even with the 2016 ACIP guideline changes recommending a two-dose vaccine series for those ages 9-14, the overall vaccination rate of only $45.4 \%$ for two doses in the NIS-Teen sample is far less than desirable. Though ACIP recommendations support two- 
doses for the young-adolescent subset, the guidelines still delineate a three-dose schedule for those ages 15-26 due to the body of research regarding two-dose immunogenicity and efficacy being conducted on those participants under the age of fifteen (Iverson et al., 2016; Meites, Kempe, \& Markowitz, 2016; Sankaranarayanan et al., 2016).

As children progress into adulthood, overall HPV vaccination rates decline. In a review of the 2014 National Health Interview Survey (NHIS), with a sample of 36,324 participants ages 19 years or greater, data findings showed that only $40.2 \%$ of women ages $19-26$ and $8.2 \%$ of men ages $19-26$ reported receiving greater than or equal to one dose of HPV vaccine (Williams et al., 2016). Some noted barriers to vaccination include status of health insurance coverage and accessibility to a consistent healthcare provider (Williams et al., 2016). With the rates of adolescent and young adult vaccinations remaining low, the population continues to be at risk for HPV-associated diseases including dysplastic tissue changes and cancer. ACIP dosing guidelines now include “catch-up” dosing schedules for older adolescents and young adults ages 13-26, and this could greatly impact the overall numbers of adults who have been vaccinated (Meites, Kempe, \& Markowitz, 2016; Williams et al., 2016). Federal programs such as the Vaccines for Children program that offers no-cost vaccination to those under the age of 19 who qualify for Medicaid, are uninsured, or underinsured, may support increased vaccination rates (CDC, 2016; Williams et al., 2016). However, health care providers should pursue alternate opportunities to vaccinate young adults, especially women who are at great risk for developing cervical cancer from HPV infection (Williams et al., 2016).

\section{Postpartum HPV Vaccination}


Pregnancy is a period of time when many women have increased access to healthcare providers who can educate about HPV and HPV vaccination (Kilfoyle, Rahangdale, \& Dusetzina, 2016). Though HPV vaccine is not recommended in pregnancy, this access provides an opportunity for health care providers to discuss the benefits of postpartum HPV vaccination and to educate patients concerning the risk of HPV.

The potential of postpartum HPV vaccination programs is dependent upon the feasibility and acceptability of postpartum vaccination programs. In a study of 150 postpartum women ages 18-26, researchers examined participants' overall acceptance of quadrivalent HPV vaccine in the postpartum period and their subsequent compliance with the three-dose series completion (Wright et al., 2012). Study participants were approached on day one or two of postpartum hospitalization. If agreeable to vaccination, participants received free-of-charge HPV vaccine while inpatient. Dose two was administered at the postpartum visit, with the third-series dose given during a subsequent follow-up appointment (Wright et al., 2012). Adherence rates through dose three were studied to determine if receiving the vaccine during the postpartum period was conducive to participants completing the series. Findings showed that $4.7 \%$ of participants did not receive any vaccines, $41.3 \%$ received one dose, $23.3 \%$ received two doses, and 30.7\% received all three doses (Wright et al., 2012). Additionally, patient satisfaction with receiving the vaccine while postpartum was surveyed, with $97.2 \%$ of participants reporting overall satisfaction with vaccination (Wright et al., 2012).

In a similar study of 500 pregnant women under the age of 26, researchers examined the likelihood of HPV vaccine acceptance in the postpartum period if the 
vaccination was offered free-of-charge (Berenson et al., 2014). The researchers also investigated barriers related to why women were not previously vaccinated (Berenson et al., 2014). Of those participants who reported that they were not previously vaccinated or who had not completed the series, $83 \%$ were willing to receive free-of-charge vaccine during the postpartum period (Berenson et al., 2014). Barriers to HPV vaccine series initiation or completion were reported as lack of prior knowledge about HPV, HPV vaccination, and safety (Berenson et al., 2014). Providing education to patients during the prenatal visit about the risk and effects of HPV, vaccination efficacy and overall safety, and administration of vaccine during the inpatient hospital stay could be an opportunity for providers to increase vaccination rates (Berenson, et al., 2014).

Despite evidence that patients are willing to accept vaccination, research suggests that postpartum vaccine initiation and series completion has been low. In a review of 48,001 health records of privately-insured female patients ages 18-26, covering the years between 2006 and 2012, 861 women received an HPV vaccine, with 337 completing the three-dose series within one year postpartum (Kilfoyle, Rahangdale, \& Dusetzina, 2016). Research findings indicate that fewer than two percent of eligible patients received the vaccine and that opportunities exist for prenatal care providers to increase patient awareness of HPV-associated disease and recommend vaccination in the postpartum period (Kilfoyle, Rahangdale, \& Dusetzina, 2016).

The evidence of high incidence and prevalence of HPV virus is irrefutable, with one in four American's being infected with the virus (CDC, 2017). Despite evidence that the immunogenic response and vaccine efficacy of HPV vaccine are positive, overall vaccine rates remain low. Regardless of recommendations by ACIP, vaccination rates 
that show only $45.4 \%$ of the targeted population have received the HPV vaccination are unsatisfactory (Reagan-Steiner et al., 2016). Research suggests that alternative opportunities to vaccinate at-risk populations should be explored (Berenson et al., 2016; Kilfoyle, Rahangdale, \& Dusetzina, 2016). With pregnancy, many women seek health promoting behaviors. HPV education and vaccination should be incorporated in the prenatal care delivery model so that patients are aware of their risks and opportunities for primary prevention (Berenson et al., 2016; Kilfoyle, Rahangdale, \& Dusetzina, 2016). 


\section{Theoretical Framework}

The Program Logic Model (PLM) was chosen as the theoretical framework to guide this project. Program evaluation encompasses more than the assessment of outcomes. To determine the effectiveness of a projected outcome, one must take methodical steps to develop activities that bring about positive transformational change (W. K. Kellogg Foundation, 1998). The Program Logic Model can guide both program development and program evaluation (W. K. Kellogg Foundation, 1998). This model utilizes a five-step approach of resource identification, activities or resource utilization, outputs or results of planned activities, outcomes or accomplishments, and impact or overall benefit to the stakeholders (W. K. Kellogg Foundation, 1998). The framework of the PLM is presented in Figure 1 below (University of Kansas, 2018).

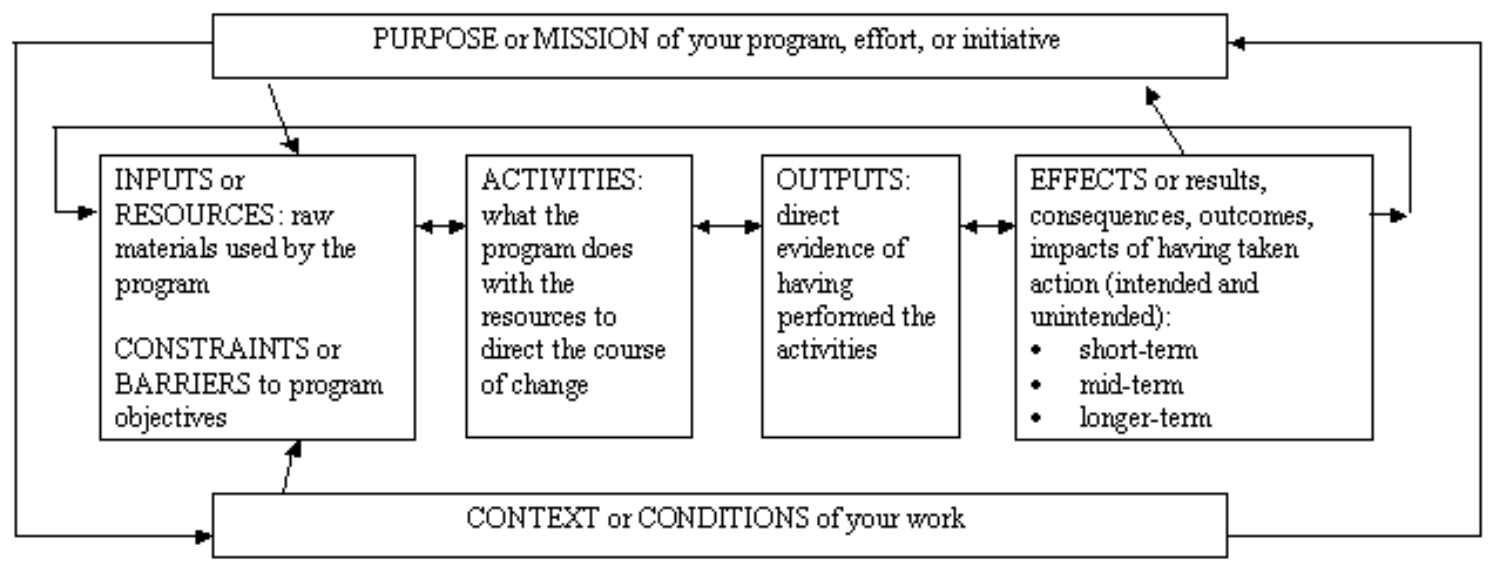

Figure 1. Program Logic Model Framework (University of Kansas, 2018).

Identification of the gap in HPV vaccination rates for young women ages 19-26

years is the beginning of the process for application of the PLM. Following the cause and effect principles of logic, one can determine if the HPV pilot vaccine program has accomplished the objective of increasing HPV vaccine uptake and completion rates in the 
targeted population. The interconnectedness of planning and implementation to the outcome should remain visible throughout the evaluation of the program's effectiveness.

With each step in the logic model comes some aspect of change, making the theory of change the theoretical underpinning of the PLM (W. K. Kellogg Foundation, 1998). Within change theory, old practices are replaced with newer more productive processes (W. K. Kellogg Foundation, 1998). Regarding the HPV vaccine program at Women and Infants Hospital, these changes include obtaining state-supplied HPV vaccine for patients ages 19-26 and introducing clinical practice changes with outpatient providers to identify HPV vaccine eligibility. Additional, process changes include inpatient nurses administering HPV vaccine to inpatient postpartum patients. Finally, adjustments to the postpartum care delivery model with the administration of HPV vaccine at the postpartum visit will be necessary. Further changes include revisions to previous workflow practices that will lead to an increased rate of overall HPV vaccine administration to Obstetrics and Gynecology Care Center patients. Both change and logic are not always linear, but should remain commonsense and be examined for their causal relationship (W. K. Kellogg Foundation, 1998). 


\section{Method}

\section{Purpose}

The purpose of this evaluation was to assess the effectiveness of a newly developed pilot program for women ages 19-26 that incorporates assessment of HPV vaccine eligibility during the prenatal period to increase uptake and completion of HPV vaccine during the postpartum period.

\section{Description of the Program}

Women and Infants Hospital is a tertiary care center in an urban area in the northeastern US which performs approximately 8,500 deliveries per year and is the ninth largest obstetrical service in the country (Care New England, 2017). The Obstetrics and Gynecology Care Center serves a large ethnically and culturally-diverse patient population, many of whom are underinsured and medically underserved.

Prior to the implementation of the pilot perinatal HPV vaccination program, assessment of the HPV vaccination status of Obstetrics and Gynecology Care Center patients was conducted at gynecologic office visits only. The implementation of this assessment prenatally, as well as the administration of the HPV vaccine during the postpartum hospitalization at Women and Infants Hospital is newly established practice that began in March 2017. The primary eligibility criterion for this pilot program is that patients must have received prenatal care at the Obstetrics and Gynecology Care Center.

The aim of the perinatal HPV vaccine program is to increase HPV vaccination uptake through the complete three-dose series for Obstetrics and Gynecology Care Center patients, ages 19-26. Patients ages 19-26 who receive prenatal care at the Women and Infants Hospital Obstetrics and Gynecology Care Center are assessed for HPV vaccine eligibility by the nurse practitioner or resident during a third-trimester office visit. 
Criteria for vaccine eligibility include a history of no previous HPV vaccination or a history of incomplete vaccine series. Additionally, because the vaccine dispensed by the hospital pharmacy is state-supplied vaccine (SSV) for adults over the age of 19, patients must be Rhode Island residents or non-Rhode Island residents who are not insured by a Rhode Island employer to receive vaccination (Rhode Island Department of Health, 2017). Contraindications include an allergy to yeast or a previous allergic reaction to HPV vaccine.

The provider documents vaccine eligibility in the electronic medical record (EMR) of those patients who have not begun or completed the HPV vaccine series, do not have contraindications, and who agree to accept the vaccine during the postpartum period. The provider documents ineligibility in the EMR of those patients who have completed the series, have an identified contraindication, or decline.

The outpatient medical record contains a text box for the provider to include a yes or no answer for vaccine eligibility. The EMR template does not require the provider to include a rationale for vaccination eligibility or ineligibility. However, a comment section has been established for the provider if they choose to document this information. The outpatient EHR template containing the HPV vaccine text box is interfaced into the inpatient EHR prior to the patient's estimated due date. In addition, all residents have access to both the outpatient and inpatient EHRs so that they may access the required prenatal assessments upon inpatient admission from labor and delivery.

The inpatient EMR templates for post-cesarean and post-vaginal delivery care includes an HPV order sentence that the provider chooses, post-delivery, for those patients who were pre-determined to be vaccine eligible during the prenatal period. The 
electronic order then populates the medication administration record with the HPV vaccine order for the nurse caring for the patient during the postpartum hospital stay. Residents and nurse practitioners who provide care at the Obstetrics and Gynecology Care Center received education during resident education sessions and Grand Rounds about the new process of screening patients during the prenatal period. Additionally, residents received further training about ordering vaccine during the inpatient period as they are the providers that deliver obstetric services to inpatient Obstetrics and Gynecology Care Center patients.

In addition to resident and nurse practitioner education, inpatient nurses on all units that provide postpartum care received education about HPV, HPV vaccination, and the new process for administering vaccine during the postpartum period. This education was in the form of a computer-based learning (CBL) module. Inpatient nurse managers and the mother-baby nurse educator were instrumental in encouraging CBL completion resulting in an 89\% completion rate for assigned nursing staff.

One dose of the three dose HPV series is administered during the inpatient postpartum period, an additional dose in the series is administered at the outpatient postpartum appointment if warranted, and the final dose, if needed to complete the vaccine series, is administered at a follow-up outpatient nurse visit six months after dose one of the series or at a minimum of twelve weeks from dose two in the series. Each Obstetrics and Gynecology Care Center patient receives a letter reminding them to schedule their postpartum visit and robocalls are placed to patients 48 and 24 hours prior to their appointment as a reminder of their follow-up visit. Prior to vaccine 
administration, vaccine information sheets are provided to each patient with each dose, as required by federal law.

Administration of SSV during the inpatient hospitalization and postpartum visit does not result in an increased cost to the patient, as SSV is free of charge to eligible patients, and the administration fee is included in the maternity insurance coverage. However, if a lapse in insurance coverage occurs after the postpartum period, the SSV will remain without cost to patients, though the possibility of a negligible administration charge for the vaccine at the nurse follow-up visit may be realized.

\section{Research Questions}

The research questions for this program evaluation were:

1. How many patients ages 19-26 received a dose of HPV vaccine after delivery while an inpatient at Women and Infants Hospital during the period of March 1, 2017 through December 31, 2017 ?

2. How many patients ages 19-26 received a dose of HPV vaccine during the postpartum period while an outpatient at the Obstetrics and Gynecology Care Center during the period of March 1, 2017 through December 31, $2017 ?$

3. How many patients ages 19-26 who received an inpatient HPV vaccine dose and a postpartum outpatient HPV vaccine dose also received an outpatient dose after the postpartum period during the period of March 1, 2017 through December 31, 2017? 
4. What percentage of HPV vaccines administered were given according to ACIP guidelines during the period of March 1, 2017 through December 31, 2017?

5. How many total HPV vaccinations were administered to Obstetrics and Gynecology Care Center patients ages 19-26 between June 1, 2016 through February 28, 2017 prior to program implementation?

6. How many total HPV vaccinations were administered to Obstetrics and Gynecology Care Center patients ages 19-26 between March 1, 2017 through December 31, 2017?

7. What is the number of Obstetrics and Gynecology Care Center patients ages 19-26 in the ten-months pre-program implementation?

8. What is the number of Obstetrics and Gynecology Care Center patients ages 19-26 in the ten-months post-program implementation?

\section{Design}

A retrospective chart review examining pre-program implementation and postprogram implementation of HPV vaccine data during June 1, 2016 through December 31, 2017 was conducted. To answer the research questions, data on the number of inpatients who received HPV vaccine between March 1, 2017 through December 31, 2017 including medical record number (MRN), date of birth, and date of vaccine administration was requested. Outpatient data, including MRN, date of birth, and date of vaccine administration, was requested on patients who had also received an inpatient HPV dose during the timeframe of March 1, 2017-December 31, 2017. 
The number of Obstetrics and Gynecology Care Center patients ages 19-26 from June 1, 2016 through February 28, 2017 and March 1, 2017 through December 31, 2017 was obtained. Also, the number of HPV vaccines given to all patients ages 19-26 at the Obstetrics and Gynecology Care Center from June 1, 2016 through February 28, 2017 and March 1, 2017 through December 31, 2017 was tracked.

\section{Sample}

The sample consisted of perinatal patients ages 19-26 who received prenatal care at the Women and Infants Hospital Obstetrics and Gynecology Care Center, were hospitalized postpartum at Women and Infants Hospital, and agreed to receive postpartum HPV vaccination.

\section{Sites}

Women and Infants Hospital in Providence, Rhode Island and the outpatient Women and Infants Hospital Obstetrics and Gynecology Care Center in Providence, Rhode Island were the locations of the study.

\section{Procedures}

A proposal for evaluating the Women and Infants Hospital Obstetrics and Gynecology Care Center perinatal vaccination pilot program was approved by both the Rhode Island College and the Women and Infants Hospital Institutional Review Boards (IRB). The IRB approval documents are available in Appendices A and B. Collaboration with the Care New England (CNE) Health System pharmacy informatics department and the Obstetrics and Gynecology Care Center Senior Division Manager was necessary for obtaining clinical data. Data on patients ages 19-26 focused on the time frames of June 1, 2016 through February 28, 2017 and March 1, 2017 through December 31, 2107 and 
included: the number of patients who received care at the Obstetrics and Gynecology Care Center; the number of HPV vaccines administered to Obstetrics and Gynecology Care Center patients; the number of vaccines administered to individual patients while inpatient at Women and Infants Hospital; and the number of vaccines administered to individual patients while outpatient at the Obstetrics and Gynecology Care Center. Data collected from the perinatal vaccination pilot program was performed retrospectively. Patient identifiers associated with the data collection including MRN and dates of birth were electronically accessed and stored through the secured CNE data server to protect patient identity.

The request to the CNE pharmacy informatics department specifically included:

1. Medical record number, date of birth, and date of injection for each patient, ages 19-26, who received a dose of HPV vaccine after delivery while an inpatient at Women and Infants Hospital between March 1, 2017 through December 31, 2017.

2. Medical record number, date of birth, and date of injection for each patient ages 19-26 who received a dose of HPV vaccine during the postpartum period while an outpatient at the Obstetrics and Gynecology Care Center between March 1, 2017 through December 31, 2017.

3. Medical record number, date of birth, and date of injection for each patient ages 19-26 who received an inpatient HPV vaccine dose, a postpartum outpatient HPV vaccine dose, and also received an outpatient dose after the postpartum period; between March 1, 2017 through December 31, 2017. 
4. Number of total HPV vaccinations were administered to Obstetrics and Gynecology Care Center patients ages 19-26 between June 1, 2016 through February 28, 2017 prior to program implementation,

5. Number of total HPV vaccinations were administered to Obstetrics and Gynecology Care Center patients ages 19-26 between March 1, 2017 through December 31, 2017.

Chart review data from the CNE pharmacy informatics department was requested in report form including MRN, date of birth, and date of vaccine administration for all patients who received an inpatient dose of HPV vaccine. Additionally, outpatient data was requested on patients who received an inpatient dose including $\mathrm{MRN}$, date of birth, and date of vaccine administration. Once patients were correlated to doses by MRN, the MRN data was electronically deleted and patients were assigned a number for identification to maintain anonymity.

The data request to the Obstetrics and Gynecology Care Center Senior Division Manager included:

1. Total number of Obstetrics and Gynecology Care Center patients ages 1926 in the ten-months pre-program implementation.

2. Total number of Obstetrics and Gynecology Care Center patients ages 1926 in the ten-months post-program implementation.

The chart review data was requested in report form as a total volume, again maintaining anonymity.

As comparative data for pre-program inpatient HPV vaccination did not exist, a comparison of the number of HPV vaccines given from the Obstetrics and Gynecology 
Care Center in the 10 months pre-program implementation to the 10 months postprogram implementation was used to assess if the program was effective at increasing uptake and completion of HPV vaccine during the postpartum period. Through a chart review, postpartum patients age 19-26 were identified and specific areas of focus were examined. These included: 1). Was an HPV dose administered to vaccine eligible individuals during the inpatient period? 2). Was an additional dose administered at an ambulatory postpartum visit? and 3) Was a subsequent dose given at an ambulatory visit if warranted?

\section{Data Analysis}

Analysis of the data was performed using MRN's to correlate patients to doses. In addition, dates of birth allowed for examination of trends in vaccination by age. Date of injection was analyzed to determine if doses were administered at appropriate intervals. Frequencies and percentages in relation to numbers of patients and HPV doses were calculated. 


\section{Results}

A total of 30 postpartum Obstetrics and Gynecology Care Center patients

received a dose of HPV vaccine while inpatient at Women and Infants Hospital between March 1, 2017 and December 31, 2017. Prior to the implementation of the program in March 2017 no patients received HPV vaccine while inpatient at Women and Infants Hospital. Table 1 shows the distribution by patient age with a mean age of 22.48 years. Table 1: Inpatient HPV Vaccines 3.1.17-12.31.17 by patient age

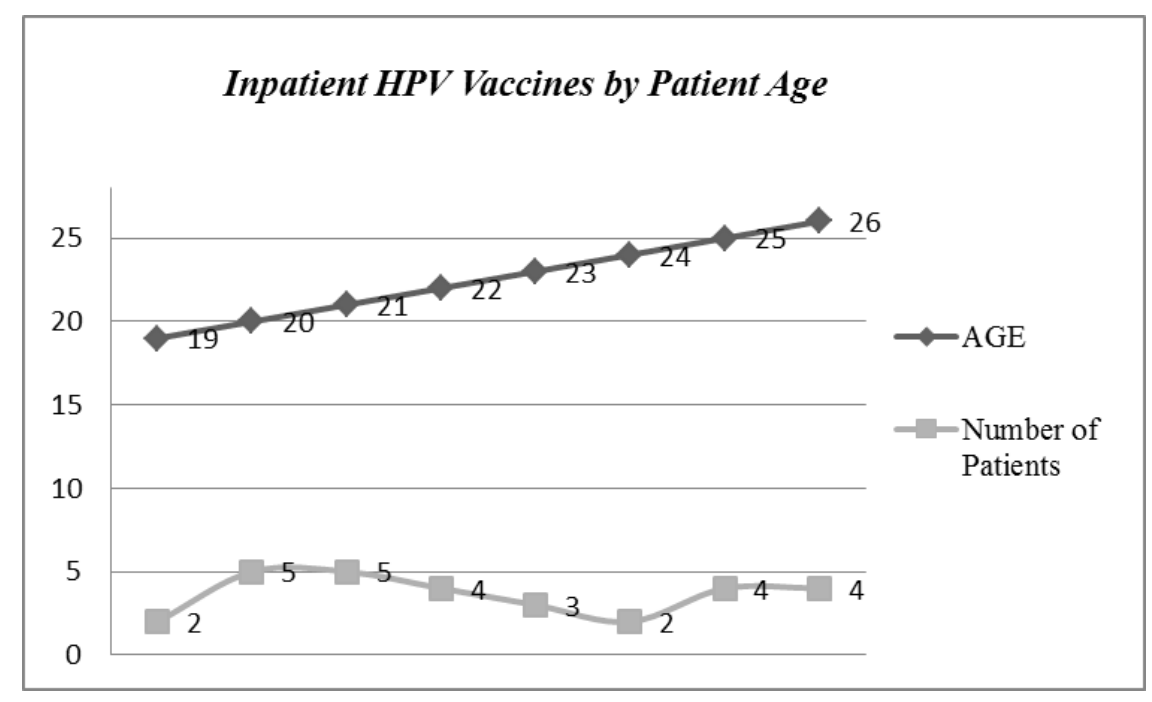

Of the 30 patients who received an inpatient dose, six patients received a dose at their outpatient postpartum visit; and two patients received a third-dose at a subsequent outpatient visit. ACIP guidelines call for minimum intervals between HPV doses: four weeks between dose one and two, twelve weeks between dose two and three, and a minimum of five months from dose one to three (CDC, 2017). Table 2 shows the vaccine intervals for the selected patients who received doses following inpatient dosing. One patient, identified as patient 4 , received a $4^{\text {th }}$ dose of HPV vaccine 13.4 weeks after completing the 3-dose series. 
Table 2: Vaccine Intervals, By Patient, Following Inpatient Dosing

Vaccine Intervals, By Patient, Following Inpatient Dosing

\begin{tabular}{|c|c|c|}
\hline Patient & $\begin{array}{l}\text { Interval from Inpatient dose } \\
\text { (weeks/days) }\end{array}$ & $\begin{array}{l}\text { Interval from Postpartum } \\
\text { dose (weeks/days) }\end{array}$ \\
\hline 1 & 5.6 & \\
\hline 2 & 7.6 & \\
\hline 3 & 5.3 & 22.6 \\
\hline 4 & 5 & 17.4 \\
\hline 5 & 6.1 & \\
\hline 6 & 7 & \\
\hline
\end{tabular}

During the period from June 1, 2016 through February 28, 2017, 1918 patients ages 19 through 26 received care at the Obstetrics and Gynecology Care Center. During the same period, seventy HPV vaccines were administered to this patient subset, representing $4 \%$ of the patient population. Comparatively, during the period from March 1, 2017 through December 31, 2017, 877 patients ages 19 through 26 received care at the Obstetrics and Gynecology Care Center. HPV vaccine given to this patient group totaled 155 doses, representing $18 \%$ of the patient population and demonstrating an increase of 14\% following initiation of the program. Table 3 shows the percentages of HPV vaccine given to the targeted age group pre-program implementation and post-program implementation. 
Table 3: Percentage of HPV Vaccine Given Pre- and Post-Program Implementation

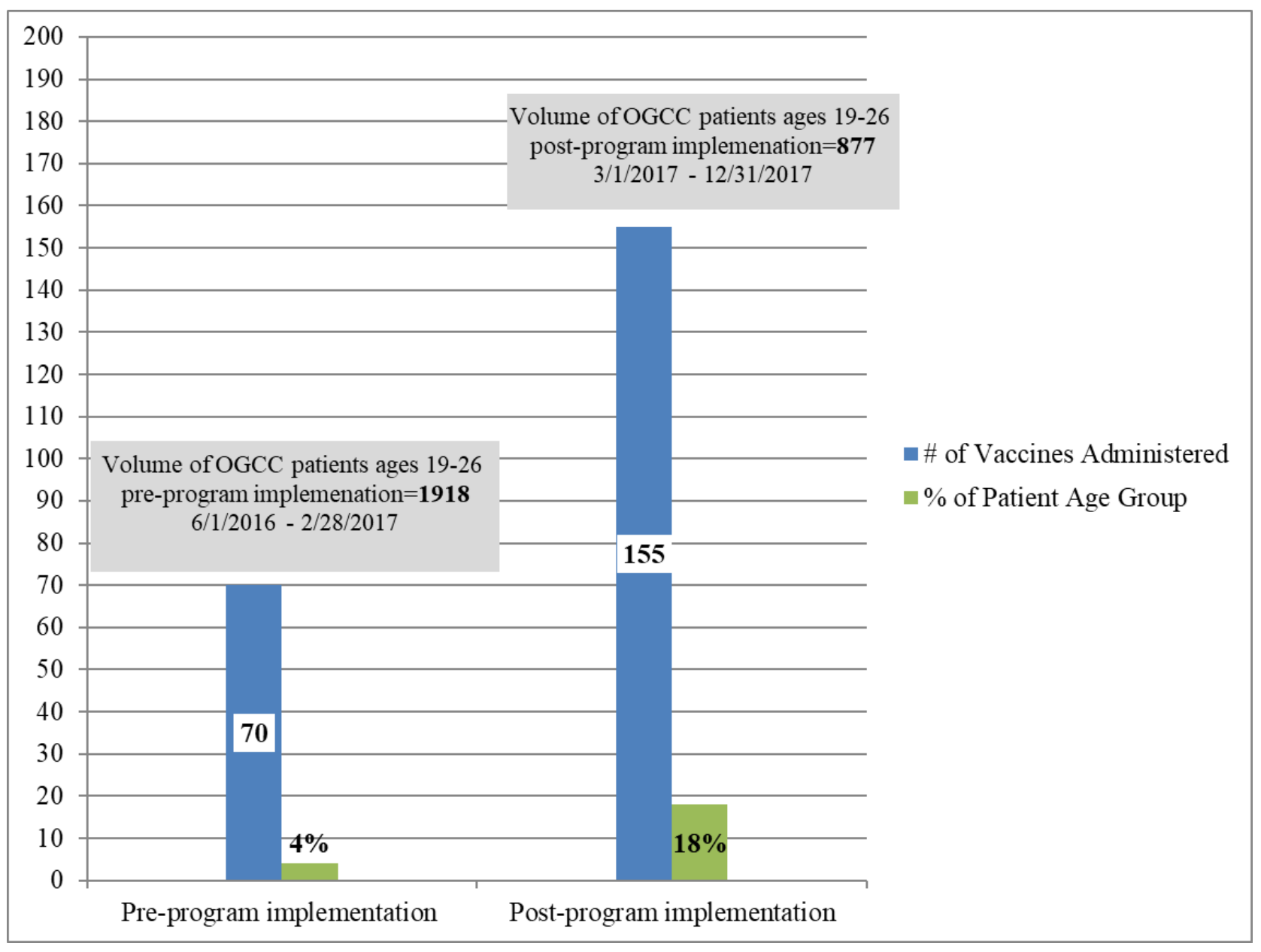




\section{Summary and Conclusions}

\section{Summary}

The purpose of this evaluation was to assess the effectiveness of a newly developed pilot program for women ages 19-26 that incorporates assessment of HPV vaccine eligibility during the prenatal period to increase uptake and completion of HPV vaccine during the postpartum period. A retrospective chart review examining preprogram implementation and post-program implementation HPV vaccine data during June 1, 2016 through December 31, 2017 was conducted. The sample consisted of perinatal patients' ages 19-26 who received prenatal care at the Women and Infants Hospital Obstetrics and Gynecology Care Center, were hospitalized postpartum at Women and Infants Hospital, and agreed to receive postpartum HPV vaccination.

A proposal for evaluating the Women and Infants Hospital Obstetrics and Gynecology Care Center perinatal vaccination pilot program was approved by both the Rhode Island College and the Women and Infants Hospital IRB. Chart review data was analyzed for all patients who received an inpatient dose of HPV vaccine. Additionally, outpatient data was requested on patients who received an inpatient dose. As comparative data for pre-program inpatient HPV vaccination did not exist, comparing the number of HPV vaccines given from the Obstetrics and Gynecology Care Center in the 10 months pre-program implementation to the 10 months post-program implementation was used to evaluate if the program was effective at increasing uptake and completion of HPV vaccine during the postpartum period.

The results of the chart review showed 30 patients who received an inpatient dose of HPV vaccine. Of these patients, six received a dose at their outpatient postpartum visit, 
and two of these patients received a subsequent dose to complete the series. Of note, the EMR where vaccine eligibility is documented does not include any fields indicating if the patient was eligible for vaccination due to being unvaccinated or if the patient had not previously completed the vaccine series. This makes determining which number vaccine in the series was given impossible except for the two patients who received three doses.

Vaccine timing is an important factor with HPV vaccine series. ACIP guidelines call for minimum intervals between HPV doses: four weeks between dose one and two, twelve weeks between dose two and three, and a minimum of five months from dose one to three (CDC, 2017). Chart review data for the six patients who received doses at their outpatient postpartum visit showed all received their dose within the appropriate interval with an average date of receipt being 6.1 weeks from their inpatient dose. Data for the two patients who received a third-dose in the series also met the ACIP minimum dosing interval for dose three. However, one patient who had received all three doses in the appropriate dosing interval received a fourth dose. The use of separate EMR systems for inpatient and outpatient documentation may have caused this discrepancy to occur.

\section{Limitations}

An examination of the number of HPV doses given to Obstetrics and Gynecology Care Center patients ages 19-26 given in the 10-months pre-program implementation and the 10 -months post-program implementation showed an overall increase of $14.02 \%$. It is difficult to determine if the vaccination increase is a result of the concurrent introduction of free of charge, to eligible patients, state supplied HPV vaccine or due to the process changes implemented to include screening of prenatal patients for vaccine eligibility. In addition, data examined was for doses of HPV vaccine given to all Obstetrics and 
Gynecology Care Center patients ages 19-26 pre and post program and did not specifically analyze data for pregnant patients alone.

\section{Conclusion}

In the evaluation of the effectiveness of the pilot program for women ages 19-26 that incorporated assessment of HPV vaccine eligibility during the prenatal period to increase uptake and completion of HPV vaccine during the postpartum period, several discoveries occurred. With the determination of vaccine eligibility, prenatally patients do express interest in being vaccinated in the inpatient setting. Without data on the overall number of pregnant patients who received care at the Obstetrics and Gynecology Care Center during the two-examined time frames, it is not possible to determine the percentage of pregnant patients who received the vaccine as inpatients or during their outpatient visits. However, the data is encouraging in that pre-program HPV vaccination was not occurring in the inpatient setting and with the introduction of this program, 30 eligible patients did receive vaccination. Prior research has shown that HPV titers increase even with one vaccine though at lower levels then the complete vaccine series (Sankaranarayanan et al., 2016). The conclusion that may be reached is that even one dose of HPV vaccine could result in a higher level of immunity than natural immunity alone, thereby decreasing the potential of HPV infection and resultant morbidity and mortality from related cancers.

Looking beyond the examined patient population of women ages 19-26, further research on strain-specific HPV infection rates in women may determine if vaccination programs that target all eligible patients results in a decrease in HPV related cancers in the future. In October, 2018, the Federal Drug Administration approved the use of 
Gardasil 9 for both men and women ages 27 through 45 years old for the prevention of vaccine specific HPV-associated cancers, dysplasias, and genital condyloma (U. S. DHHS, 2018). Notably, though expanded HPV vaccination has received FDA approval, insurance carriers have yet to reach a universal determination on vaccine cost or coverage to the direct consumer. 


\section{Recommendations and Implications for Advanced Nursing Practice}

In the United States, seventy-nine million Americans, or one in four, are infected with HPV (CDC, 2017). By targeting HPV strains that are oncogenic and related to genital condyloma, HPV vaccine could be revolutionary in combating the most prevalent sexually transmitted infection worldwide. Though vaccine efficacy is greatest when introduced prior to sexual debut, evidence supports that HPV vaccination for eligible patients through the age of 26 may be beneficial in offering protection against HPV strains not previously acquired and could significantly impact the rates of HPVassociated cancers in women (Serrano et al., 2012).

As the prevalence of HPV infection is widespread, on a macro-level, our entire global society becomes stakeholders in the vaccination debate which has significant implications for clinical practice. With a narrower focus, those greatly impacted are the children of today who are at-risk for HPV infection due to sexual exposure in the future. Additional stakeholders, on both a macro and micro-level, include individual vaccine recipients, parents, healthcare providers, healthcare systems, and policy makers. Providing evidence-based education to stakeholders, increasing vaccine accessibility to all eligible populations including adults through age 26, and seeking alternate options for HPV vaccination beyond the primary care providers office is crucial.

With most HPV vaccine programs targeting adolescents, opportunities exist to examine those that support and promote the vaccination of other eligible populations. Increasing human papillomavirus (HPV) vaccination rates can be considered a priority based on the major drivers of public health quality. Those drivers include populationcentered services that prevent and control disease (Honoré et al., 2011). In addition, public health quality programs must fundamentally be proactive, health-promoting, and 
risk-reducing in that they aim to mitigate current and emerging disease, increase positive outcomes, and prevent illness or negative results (U.S. DHHS, 2008). HPV vaccination programs align with the aims in that vaccination before sexual debut is proactive in preventing HPV, health-promoting as it targets reduction of HPV in society, and riskreducing in its ability to prevent HPV-associated sequalae.

Public policy surrounding HPV vaccination has and continues to be a controversial topic . With many states enacting legislation to support, mandate, and provide access to HPV vaccine. Many others have fought to minimize governmental involvement in HPV vaccination or expand legislation that allows for parents to "opt out" for moral or philosophical reasons. It is important that policies that require HPV vaccination expand beyond our state and our nation and expand to our global community. HPV-associated cancers are preventable through vaccination. Not only should children on the state level be afforded the opportunity to live without HPV-associated disease, but this same opportunity should be supported, promoted, and encouraged by national and worldwide policy makers.

Development and promotion of HPV vaccination programs is a core goal of public health nursing practice as nurses promote and protect the health of populations (American Public Health Association, 2013). "Population-focused nursing integrates the tenets of public health with clinical care, thus contributing to improving the health of communities and population” (Storfjell, Winslow, \& Saunders, 2017). Developing a program for HPV vaccination involves a variety of nursing interventions including: developing policy; making HPV vaccination standard practice and equitably available; advocating for and promoting HPV primary-prevention programs; and educating the 
community at-large who are all significantly affected by the negative impact of HPV. Opponents of HPV vaccination have voiced their concern due to ethical, religious, moral, and political reasons. While proponents have supported the vaccine's efficacy, economic savings, and societal impact in reducing overall HPV rates and the long-term sequelae associated with HPV infection.

Further research is necessary to continue to reduce the prevalence of HPV in society and decrease the burden of disease associated with this preventable virus. As HPV vaccine is relatively young in comparison to many childhood immunizations new and emerging data on vaccine efficacy continues to lead to practice changes in HPV vaccination. This can be seen currently in the reduction of the vaccine series from three doses to two doses for certain populations under age 15. With expanded research, barriers, attitudes, and accessibility to HPV vaccination should be explored. Additionally, further research on 2-dose vaccine series for additional populations should be investigated. Nurses are poised to make a significant impact on women's' health as they seek innovative ways to introduce evidence-based research and practice to improve the health of the population. 


\section{References}

American Public Health Association. (2013). The definition and practice of public health nursing. Retrieved from

https://www.apha.org/ /media/files/pdf/membergroups/phn/nursingdefinition.ash $\mathrm{X}$

Berenson, A. B., Male, E., Lee, T. G., Barrett, A., Sarpong, K. O., Rupp, R. E., \& Rahman, M. (2014). Assessing the need for and acceptability of a free-of-charge postpartum HPV vaccination program. American Journal of Obstetrics \& Gynecology, 210(3), 213.e1-213.e7. doi: 10.1016/j.ajog.2013.11.036

Braaten, K. \& Laufer, M. (2008). Human papillomavirus (HPV), HPV-related disease, and the HPV vaccine. Reviews in Obstetrics and Gynecology, 1(1), 2-10.

Retrieved from https://www.ncbi.nlm.nih.gov/pmc/articles/PMC2492590/pdf/RIOG01001_0002. pdf

Burdette, A. M., Webb, N. S., Hill, T. D., \& Jokinen-Gordon, H. (2017). Race-specific trends in HPV vaccinations and provider recommendations: persistent disparities or social progress? Public Health, 142, 167-176. doi:

http://dx.doi.org/10.1016/j.puhe.2016.07.009

Centers for Disease Control and Prevention. (2014, October). Advisory Committee on Immunization Practices (ACIP) summary report. Retrieved from https://www.cdc.gov/vaccines/acip/meetings/downloads/min-archive/min-201410.pdf 
Centers for Disease Control and Prevention. (2016). Vaccines for children program (VFC). Retrieved from https://www.cdc.gov/vaccines/programs/vfc/about/index.html

Centers for Disease Control and Prevention. (2017). HPV and cancer: How many cancers are linked with HPV each year? Retrieved from https://www.cdc.gov/cancer/hpv/statistics/cases.htm

Centers for Disease Control and Prevention. (2017). Recommended immunization schedule for adults aged 19 years or older, by vaccine and age group. Retrieved from https://www.cdc.gov/vaccines/schedules/hcp/imz/adult.html de Sanjose, S., Quint, W. G., Alemany, L., Geraets, D. T., Klaustermeier, J. E., Lloveras, B., ... Bosch, F. X. (2010). Human papillomavirus genotype attribution in invasive cervical cancer: A retrospective cross-sectional worldwide study. Lancet Oncology, 11(11), 1048-1056. doi: http://dx.doi.org/10.1016/S14702045(10)70230-8

Handler, N., Handler, M., Majewski, S., \& Schwartz, R. (2015). Human papillomavirus vaccine trials and tribulations: Vaccine efficacy. Journal of the American Academy of Dermatology, 73(5), 759-767. doi: http://dx.doi.org/10.1016/j.jaad.2015.05.040

Honoré, P. A., Wright, D., Berwick D. M., Clancy, C. M., Lee, P., Nowinski, J., \& Koh, H. K. (2011). Creating a framework for getting quality into the public health system. Health Affairs, 30(4), 737-745. doi: 10.1377/hlthaff.2011.0129

Iversen, O., Miranda, M, J., Ulied, A., Soerdal, T., Lazarus, E., Chokephaibulkit, K., ... Luxembourg, A. (2016). Immunogenicity of the 9-valent HPV vaccine using 2- 
Dose Regimens in Girls and Boys vs a 3-Dose Regimen in Women. Journal of the American Medical Association. 316(22), 2411-2421. doi:

10.1001/jama.2016.17615

Joura, E., Giuliano, A., Iversen, O., Bouchard, C., Mao, C., Mehlsen, J., ... Luxembourg, A. (2015). A 9-Valent HPV vaccine against infection and intraepithelial neoplasia in women. New England Journal of Medicine, 372(8), 711-723.

Kilfoyle, K., Rahangdale, L., \& Dusetzina, S. (2016). Low uptake of human papillomavirus vaccine among postpartum women, 2006-2012. Journal of Women’s Health, 25(12), 1256-1261. doi:10.1089/jwh.2016.5834

Meites, E., Kempe, A., \& Markowitz L. (2016). Use of a 2-Dose Schedule for Human Papillomavirus Vaccination -Updated Recommendations of the Advisory Committee on Immunization Practices. Morbidity and Mortality Weekly Report, 65(49), 1405-1408. doi: http://dx.doi.org/10.15585/mmwr.mm6549a5.

National Cancer Institute. (2016). Human papillomavirus (HPV) vaccines. Retrieved from https://www.cancer.gov/about-cancer/causes-prevention/risk/infectiousagents/hpv-vaccine-fact-sheet

Petrosky, E., Bocchini J., Hariri, S., Chesson, H., Curtis, C. R., Saraiya, M., ... Markowitz, L. (2015). Use of 9-valent human papillomavirus (HPV) vaccine: Updated HPV vaccination recommendations of the Advisory Committee on Immunization Practices. Morbidity and Mortality Weekly Report, 64(11), 300304.

Reagan-Steiner, S., Yankey, D., Jeyarajah, J., Elam-Evans, L., Curtis, C. R., MacNeil, J., ... Singleton, J. (2016). National, regional, state, and selected local area 
vaccination coverage among adolescents aged 13-17 years-United States, 2015. Morbidity and Mortality Weekly Report, 65(33), 850-858. Retrieved from https://www.cdc.gov/mmwr/volumes/65/wr/mm6533a4.htm

Rhode Island Department of Health. (2017). Immunization information for healthcare providers. Retrieved from http://www.health.ri.gov/immunization/for/providers/

Rhode Island Health and Human Services. (2017). RIte care. Retrieved from http://www.eohhs.ri.gov/Consumer/FamilieswithChildren/RIteCare.aspx

Sankaranarayanan, R., Ramesh Prabhu, P., Pawlita, M., Gheit, T., Bhatla, N., Muwonge, R., ... Pillai, M. (2016). Immunogenicity and HPV infection after one, two, and three doses of quadrivalent HPV vaccine in girls in India: A multicenter prospective cohort study. Lancet Oncology, 17, 67-77. doi: http://dx.doi.org/10.1016/S1470-2045(15)00414-3

Saraiya, M., Unger, E. R., Thompson, T. D., Lynch, C. F., Hernandez, B.Y., Lyu, C.W., ...Goodman, M. T. (2015). US assessment of HPV types in cancers: Implications for current and 9-valent HPV vaccines. Journal of the National Cancer Institute, 107(6), 1-12. doi: 10.1093/jnci/djv086

Serrano, B., Alemany, L., Tous, S., Bruni, L., Clifford, G. M., Weiss, T., ... de Sanjosé, S. (2012). Potential impact of a nine-valent vaccine in human papillomavirus related cervical disease. Infectious Agents and Cancer, 7(38), 2-13. http://doi.org/10.1186/1750-9378-7-38

Storfjell, J. L., Winslow, B. W., \& Saunders, J. S. D. (2017). Catalysts for change: Harnessing the power of nurses to build population health in the 21st century. 
Retrieved from https://www.rwjf.org/en/library/research/2017/09/catalysts-forchange--harnessing-the-power-of-nurses-to-build-population-health.html United Nations. (2015). Transforming our world: The 2030 agenda for sustainable development. Retrieved from https://sustainabledevelopment.un.org/post2015/transformingourworld/publicatio n

United States Department of Health and Human Services. (2008). Consensus statement on quality in the public health system. Retrieved from https://www.hhs.gov/ash/initiatives/quality/quality/phqf-consensusstatement.html

United States Department of Health and Human Services. (2017). HealthyPeople 2020: Immunization and infectious diseases. Retrieved from https://www.healthypeople.gov/2020/topics-objectives/topic/immunization-andinfectious-diseases/objectives

United States Department of Health and Human Services. (2017). HealthyPeople 2020: Sexually transmitted diseases. Retrieved from https://www.healthypeople.gov/2020/topics-objectives/topic/sexually-transmitteddiseases/objectives

United States Department of Health and Human Services. (2018). Vaccines, blood, and biologics: Gardasil 9. Retrieved from https://www.fda.gov/BiologicsBloodVaccines/Vaccines/ApprovedProducts/ucm4 26445.htm 
University of Kansas. (2018). Community Tool Box. Retrieved from https://ctb.ku.edu/en/table-of-contents/overview/models-for-community-healthand-development/logic-model-development/main

W. K. Kellogg Foundation. (1998). W.K. Kellogg Foundation Logic Model Development Guide. Retrieved from https://www.wkkf.org/resourcedirectory/resource/2006/02/wk-kellogg-foundation-logic-model-developmentguide

Williams, W., Lu, P., O’Halloran, A., Kim, D. K., Grohskopf, L, A., Pilishvili, T., ... Bridges, C. B. (2016). Surveillance of vaccination coverage among adult populations-United States, 2014. Morbidity and Mortality Weekly Report, 65(1), 1-36. doi: http://dx.doi.org/10.15585/mmwr.ss6501a1.

World Health Organization. (2016). Guide to introducing HPV vaccine into national immunization programmes. Retrieved from http://www.who.int/immunization/diseases/hpv/HPV_vaccine_intro_guide_draft_ Oct2016.pdf?ua=1

World Health Organization. (2017). Immunization, vaccines and biologicals: Human papillomavirus (HPV). Retrieved from http://www.who.int/immunization/diseases/hpv/en/

Wright, J., Govindappagari, S., Pawar N., Cleary, K., Burke, W., Devine, P., ... Herzog, T. (2012). Acceptance and compliance with postpartum human papillomavirus vaccination. Obstetrics \& Gynecology, 120(4), 771-782. doi:

10.1097/AOG.0b013e31826afb56 


\section{Appendix A}

-1 -

DATE: February 28, 2018

Project No. WIH 17-0108

101 Dudley Street

Providence, Rhode Island 02905

401-274-1100

womenandinfants.org

LETTER OF APPROVAL

PROJECT TITLE: [1133642-2] EVALUATION OF A PERINATAL HUMAN PAPILLOMAVIRUS

VACCINATION PROGRAM

TO: Lauren Thorngate

FROM: Care New England - Women \& Infants IRB (IRB00000746)

SUBMISSION TYPE: Response/Follow-Up

ACTION: APPROVED

APPROVAL DATE: February 28, 2018

EXPIRATION DATE: January 25, 2019

EXPEDITED REVIEW: In accordance with 45 CFR 46.110(b)(1), Category 5

Thank you for submitting the Response/Follow-Up materials for this project. The Care New England Women \& Infants IRB has APPROVED your submission. The IRB conducted a risk-benefit analysis, and determined the research falls into the category "Minimal Risk." This approval is based on an appropriate risk/benefit ratio and a project design wherein the risks have been minimized. All research must be conducted in accordance with this approved submission.

The following items are acknowledged in this submission:

- Care New England - Research Application - Care New England - Research Application (UPDATED:

$02 / 5 / 2018$ )

- Other - Administrative Report Form (UPDATED: 02/16/2018)

- Protocol - Retrospective Protocol track changes (UPDATED: 02/16/2018)

- Protocol - Retrospective Protocol.clean copy (UPDATED: 02/16/2018)

This submission has received Expedited Review based on applicable federal regulations. It is the responsibility of the principal investigator to ensure that the study is conducted as approved by the IRB. All protocol modifications/changes must be approved by the IRB before any changes are implemented except when necessary to eliminate immediate hazard to subjects. You are required by federal regulations and hospital policy to immediately report any unanticipated problems untoward effects or reactions, serious side effects and/or deaths of subjects involved and related to this project to the IRB.

If written informed consent is required: the consent form(s) have been stamped and are available under Board Documents in IRBNet. Please review the consent form(s) to be sure they are correct and are stamped with IRB approval and expiration dates. The consent form(s) and other study documents included in this submission are to be used immediately for this study and void any previously issued documents, if applicable.

IRB approval for this project expires on January 25,2019 . If you wish to continue your research after this date, you are required to submit a continuing review form 45 days prior to expiration of approval. Federal regulations do not allow for any grace period for renewal.

- 2 - Generated on IRBNet

Please provide a study closure report to the IRB when research is completed. The study closure submission form (SUBMISSION FORM: Continuing Review or Closure Report) as well as all other forms (e.g., amendment/modification, event reporting, etc.) are located in the Forms and Templates Library in IRBNet.

NO RESEARCH DATA SHOULD EVER BE STORED ON A LAPTOP. This includes data from chart reviews, surveys, and all types of research. Obtaining consent and research authorization does not 
remove the requirements and restrictions of the HIPAA security rule. For more information contact IRB Administration.

This institution is in compliance with the ICH GCP as they correspond to the FDA/DHHS regulations.

This document has been electronically signed in accordance with all applicable regulations, and a copy is retained within our records. 


\section{Appendix B}

The proposal for the project referenced below has been APPROVED by the Institutional Review Board (IRB).

Project title: Evaluation of a Perinatal Human Papillomavirus Vaccination Program

Approval \#: 1718-1583

Type of review: Expedited

Proposal type: Original

Principle Investigator: Byrd, Mary

Fees received: 1. No fees -- RIC supervised or sponsored

Funding status:

Approval date: $3 / 14 / 2018$

Expiration date: 3/14/2019

A request to renew this protocol must be received by $2 / 14 / 2019$.

Click here to access the protocol:

https://ric.topazti.net/RIC/SL/Default.aspx?linkParms=NPqkQNfZcnVbDZabLuFdTg\%3d\% $\underline{3 \mathrm{~d}}$

Your responsibilities as the Principal Investigator on this project are as follows:

1. You may implement only those materials and methods approved by the IRB. Changes to the protocol topic or methods, including the elimination of previously-approved methods, require prior approval.

2. If you are using signed consent materials, a PDF of the form(s) with the approval stamp will be uploaded to your protocol. You must use this copy with participants.

3. Unanticipated problems or adverse events must be reported within three (3) days of your knowledge of the event.

4. If you wish to continue the project beyond the expiration date, you must complete and submit a progress/final report within 30-days prior to the expiration date. If approval to continue the project is not finalized by the expiration date, you must discontinue all work pertaining to this protocol and wait until approval is given before resuming data collection. 
5. You must keep all research data and consent documents within your possession in a secured location for at least three (3) years after the completion of the study, including publications or presentations of any reports.

Do not reply to this "RIC_Elements" email address because it will not be received by the IRB. Send all correspondence to IRB@ric.edu.

Best Regards,

Cindy Padula, Ph.D.

Professor

Chair, IRB

Rhode Island College

IRB@ric.edu 